\title{
Interface-induced conversion of infrared to visible light at semiconductor interfaces
}

\author{
F. A. J. M. Driessen,* H. M. Cheong, A. Mascarenhas, and S. K. Deb \\ National Renewable Energy Laboratory, 1617 Cole Boulevard, Golden, Colorado 80401 \\ P. R. Hageman, G. J. Bauhuis, and L. J. Giling \\ Department of Experimental Solid State Physics, Research Institute for Materials, University of Nijmegen, Toernooiveld, \\ 6525 ED Nijmegen, The Netherlands \\ (Received 8 April 1996)
}

\begin{abstract}
Efficient, low-temperature conversion of infrared light into visible light (red, orange, green) is reported at single heterojunctions and undoped quantum wells of $\mathrm{GaAs}$ and ordered $\mathrm{Al}_{x} \mathrm{Ga}_{1-x} \mathrm{InP}_{2}$; an increase in photon energy of $700 \mathrm{meV}$ is obtained. The signal originates from the high-band-gap layers and disappears only if the excitation energy is tuned below the GaAs band gap. The intensity of the up-converted photoluminescence (PL) is found to decrease significantly slower with increasing temperature than that of the regular PL and it remains observable up to $200 \mathrm{~K}$. Interface-induced cold Auger processes along with the presence of trapped states for both electrons and holes in these ordered alloys account for this nonlinear mechanism. A colinear double-beam experiment confirms this. [S0163-1829(96)50132-3]
\end{abstract}

Up-conversion is the observation of radiative transitions at higher energies than that of the excitation source. In general, the observation of up-conversion requires either high temperatures to achieve, for example, (i) a thermal population of holes for momentum (k) conserving bulk Auger processes, or (ii) a thermal population of phonon modes for anti-Stokes Raman lines, ${ }^{1}$ or it relies on a nonlinear mechanism, such as two-photon absorption via real or virtual states. In addition, the excited carriers should not immediately relax back to their ground states to enable the observation of radiative transitions from the excited state. Experimental observation of up-conversion at cryogenic temperatures (cold up-conversion) in solids was reported only rarely and they concerned only small energy gains or high excitation densities. $^{2-5}$ Recently, Kim et al. ${ }^{6}$ observed up-conversion ("anti-Stokes luminesence") in an asymmetric doublequantum-well system, which they attributed to quantum oscillations.

In a recent theoretical paper, Zegrya and Kharchenko ${ }^{7}$ calculated that the presence of a heteroboundary substantially affects electron-electron interaction; as a result of the presence of the interface, the k-conservation requirement in the direction perpendicular to the interface is lifted. This allows channels for Auger recombination without a thermal threshold and the fast Auger particles are ejected predominantly in directions perpendicular to the heteroboundary. ${ }^{7}$ They showed that this new Auger mechanism is the dominant nonradiative decay process for GaAs interfaces even at room temperature, which illustrates the importance of the nonlinear effect. Seidel, Titkov, and co-workers ${ }^{8}$ experimentally verified ("Auger fountain'" effect) the high-efficiency up-conversion resulting from the cold Auger effect described by Zegrya and Kharchenko at InP/AlInAs ${ }_{2}$ heterojunctions (HJ's) with their type-II band offset. They observed photoluminescence (PL) from the InP at $1.42 \mathrm{eV}$ for laser energies between the interface transition of $1.23 \mathrm{eV}$ and the InP band gap. No up-conversion from their AlInAs ${ }_{2}$ was observed. In a recent paper, ${ }^{9}$ we reported efficient, low-temperature up- conversion at type-I GaAs/GaInP ${ }_{2}$ interfaces with an increase in photon energy of $400 \mathrm{meV}$. We proposed that cold Auger processes along with the presence of localization of both electrons and holes in the high-band-gap material were responsible for this radiative transition: this localization prevents the diffusion of carriers back to the GaAs.

In this paper we present up-conversion on various undoped $\mathrm{GaAs} / \mathrm{Al}_{x} \mathrm{Ga}_{1-x} \mathrm{InP}_{2}$ heterostructures and demonstrate photon-energy gains of up to $700 \mathrm{meV}$ covering the red, orange, and green regions of the spectrum. Furthermore, the temperature dependence of the up-converted photoluminescence (UPL) signal along with results from a colinear double-beam experiment agree with the proposed generation mechanism based on cold Auger processes.

The up-conversion was measured on various samples with GaAs- $\mathrm{Al}_{x} \mathrm{Ga}_{1-x} \mathrm{InP}_{2}$ interfaces. The epilayers were grown on (100) GaAs substrates misoriented $2^{\circ}$ toward [110] by metal-organic vapor-phase epitaxy at a pressure of 20 mbar with $\mathrm{AsH}_{3}, \mathrm{PH}_{3}$, trimethylgallium, trimethylaluminium, and solution-trimethylindium as precursors. Sample 1 consisted sequentially of a $1.0-\mu \mathrm{m}$ undoped $\mathrm{GaInP}_{2}$ layer, an undoped 30-nm-wide quantum well (QW) of GaAs, and a 100-nm undoped $\mathrm{GaInP}_{2}$ top layer. Sample 2 was similar but with the barrier layers replaced by $\mathrm{Al}_{0.4} \mathrm{Ga}_{0.6} \mathrm{InP}_{2}$. Sample 3 contained only a single heterointerface: $1 \mu \mathrm{m}$ of GaAs capped by $1 \mu \mathrm{m}$ of $\mathrm{GaInP}_{2}$. Finally, sample 4 consisted of a GaAs buffer layer followed by a 1.5 - $\mu$ m-thick $\mathrm{Al}_{0.2} \mathrm{Ga}_{0.8} \mathrm{InP}_{2}$ layer (see Ref. 10 for characterization of the growth series sample 4 was taken from). A temperature of $720^{\circ} \mathrm{C}$ and a V/III ratio of 125 were used during growth except for sample 4, which was grown at $760{ }^{\circ} \mathrm{C}$.

The optical experiments were performed in a closed-cycle He cryostat with a minimum temperature of $8 \mathrm{~K}$. Optical excitation for $\mathrm{PL}$ was provided by an $\mathrm{Ar}^{+}$laser, and for up-conversion and PL excitation spectroscopy (PLE), by either a dye laser with DCM special dye (600-700 nm; 1.77$2.07 \mathrm{eV}$ ), or by a tunable Ti-sapphire laser (700-860 nm; $1.45-1.77 \mathrm{eV})$. To make sure that only photons with the 


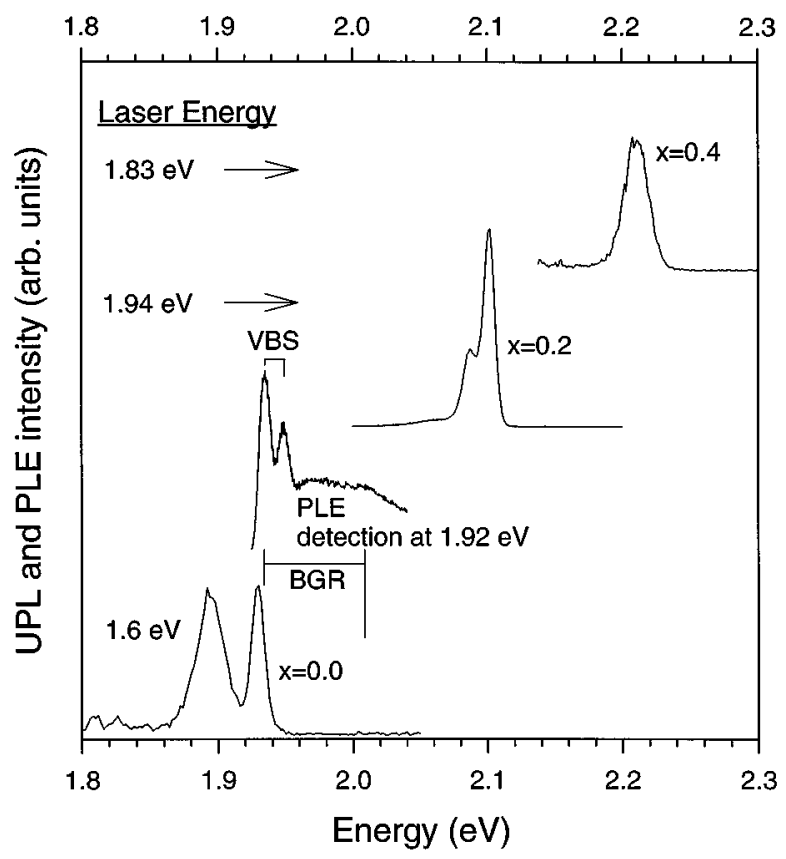

FIG. 1. Up-converted photoluminescence spectra from samples 1, 4, and 3; laser energies are indicated. The signals cover the red, orange, and green part of the spectrum. The photoluminescence excitation spectrum of sample 1 shows a pronounced valence-band splitting (VBS) of $14 \mathrm{meV}$ and a band-gap reduction (BGR) of 77 $\mathrm{meV}$ as a result of long-range ordering.

energy of the laser light reached the samples, the beam was directed through a prism monochromator, and appropriate long-pass filters were used. During PLE, the laser power was kept constant using a laser-power controller. The luminescence was dispersed by a $0.85-\mathrm{m}$ double monochromator with 1800 lines/mm gratings and detected by a Peltier-cooled photomultiplier tube with a GaAs photocathode.

Figure 1 shows UPL from the barrier layers from samples $1(x=0, \mathrm{QW}), 2(x=0.4, \mathrm{QW})$, and $4(x=0.2, \mathrm{HJ})$ with laser excitation at far lower energies, as indicated in the graph. It is seen that red $(x=0)$, orange $(x=0.2)$, and green $(x=0.4)$ up-conversion is readily obtained by varying the Al-fraction $x$ of the barrier layer(s). The PLE spectrum of sample 1 is also presented in Fig. 1 with detection at $1.92 \mathrm{eV}$ of the regular PL. This PLE spectrum shows two distinct excitonic features that originate from a splitting of the valence bands resulting from spontaneous long-range ordering in $\left(\mathrm{Ga}_{1+\eta} \mathrm{In}_{1-\eta} \mathrm{P}\right)_{1}\left(\mathrm{Ga}_{1-\eta} \mathrm{In}_{1+\eta} \mathrm{P}\right)_{1}$ monolayer super-lattices along [1 $1 \overline{1} 1]$ and [ $1 \overline{1} 11]$, where $\eta$ is the ordering parameter. From this valence-band splitting of $14 \mathrm{meV}$ and the band-gap reduction of $77 \mathrm{meV}$ as compared with the fully random alloy, which has a band gap of $2.012 \mathrm{eV}$, a value $\eta=0.4$ is calculated according to the method outlined in Refs. 11,12 .

Although junctions of $\mathrm{GaAs} / \mathrm{GaInP}_{2}$ may have only a small conduction band offset of $30 \mathrm{meV}$ (Ref. 13) so that a small fraction of the electrons might be able to overcome this barrier thermally, this is by no means the case for our GaAs/ $/ \mathrm{Al}_{x} \mathrm{Ga}_{1-x} \mathrm{InP}_{2}$ junctions, which have much larger type-I band offsets. As such, the observation of upconversion in these barrier layers is important with respect to the generation mechanism.

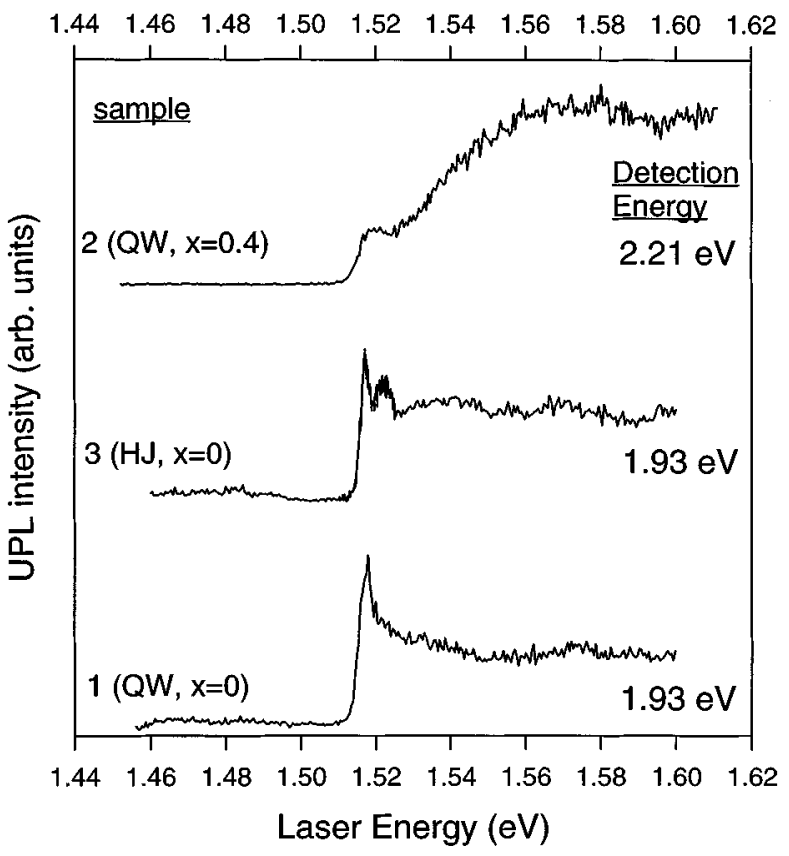

FIG. 2. UPLE spectra of samples 1, 2, and 3 showing a quenching of the UPL signal if the laser energy is tuned below the GaAs band gap for both undoped quantum wells and single heterojunctions.

In Fig. 2 the intensity of the UPL is recorded while scanning the energy of the exciting laser. These spectra show that the UPL signal disappears if the exciting laser energy is tuned below the band gap of GaAs. Furthermore, significant enhancements of the signals are observed at the energy of the GaAs exciton $(1.515 \mathrm{eV})$. These data are similar to those of our center-doped QW structure $^{9}$ and the middle graph in Fig. 2 proves that the presence of one interface is sufficient to generate the up-conversion. For sample 2 it follows that by exciting at $1.515 \mathrm{eV}$ an increase in photon energy as large as $700 \mathrm{meV}$ is achieved (conversion of $818-\mathrm{nm}$ into $560-\mathrm{nm}$ light). The sharp cutoff at the GaAs excitonic edge shows that the nonlinear process is generated by the formation of electrons and holes in the low-band-gap material. Hence, the process is interface induced and excludes as its cause all nonlinear processes in the bulk of the alloys.

The observation of interface-induced PL above the laser pump energy in type-I heterojunctions requires the presence of both up-converted electrons and holes that both do not diffuse to the GaAs. Before discussing nonlinear generation processes, we first discuss the nature of the localization mechanism that prevents this carrier diffusion. A natural explanation for the source of this is provided by the theory of Mäder and Zunger, ${ }^{12}$ predicting the existence/absence of electron/hole localization in alloys due to local clustering of the components, i.e., short-range order (SRO) with SRO parameter $\alpha>0$. Table I shows their predictions for three alloy systems: In clustering in $\mathrm{Ga}_{x} \mathrm{In}_{1-x} \mathrm{P}$ localizes holes while $\mathrm{Ga}$ clustering localizes electrons, etc. This table shows that clustering leads to both hole and electron localization only in $\mathrm{Ga}_{x} \mathrm{In}_{1-x} \mathrm{P}$ alloys while in the other alloys localization exists for either one carrier type $\left(\mathrm{Al}_{x} \mathrm{In}_{1-x} \mathrm{As}\right)$ or for none. The last column of Table I shows that this correlates with the existence/nonexistence of up-conversion at the interface with 
TABLE I. Predicted carrier localization vs observed upconverted photoluminescence (UPL) in alloys.

\begin{tabular}{llcc}
\hline \hline & \multicolumn{2}{l}{ Predicted localization } & \\
Alloy & Hole & Electron & Observed UPL \\
\hline $\mathrm{Al}_{x} \mathrm{Ga}_{1-x} \mathrm{As}$ & None & None & $\mathrm{No}^{\mathrm{a}}$ \\
$\mathrm{Ga}_{x} \mathrm{In}_{1-x} \mathrm{P}$ & on In & on Ga & Yes $^{\mathrm{a}, \mathrm{b}}$ \\
$\mathrm{Al}_{x} \mathrm{In}_{1-x} \mathrm{As}$ & on In & None & $\mathrm{No}^{\mathrm{c}}$ \\
\hline \hline
\end{tabular}

${ }^{\mathrm{a}}$ See Ref. 9.

${ }^{\mathrm{b}}$ See Ref. 9 and this paper.

${ }^{\mathrm{c}}$ See Ref. 8.

these alloys. At high growth temperatures SRO disappears, and with it the carrier localization. This too is consistent with the absence of up-conversion in high-temperature grown GaInP $_{2}$ samples. ${ }^{9}$ This analysis also suggests that upconversion can be controlled via the degree of SRO frozen into the alloy during its growth.

We now proceed with the mechanism that generates the nonlinear process. That both up-converted electrons and holes are required makes a two-step absorption process via a state at the interface highly unlikely since then only one of the carrier types would be up-converted unless both electron and hole trap states are present at the interface. Upconversion of both carrier types is, however, an inherent property for the cold interface-Auger process: the excess energy of a nonradiative electron-hole recombination in the GaAs can be used to create either a fast Auger electron or a hole (eeh and ehh processes, respectively).

For completeness, we investigated the possibility of another two-step process, where one photon generates an electron-hole pair in the GaAs (energy $E_{1}>1.52 \mathrm{eV}$ ) and the energy of a second photon is used to transfer the electron or hole across the conduction- or valence-band offsets $\left(\Delta E_{c, v}\right)$, respectively. In the case of this intrinsic two-step photon-carrier interaction, the energy of the second photon $E_{2}$ would require a value of only the maximum of $\Delta E_{c}$ and $\Delta E_{v}(\sim 0.5 \mathrm{eV})$, and an enhancement of the UPL signal generated by $E_{1}$ photons would be expected if such $E_{2}$ photons were "switched on." A two-beam experiment was performed in which the dye laser was at an energy $E_{1}$ between the low and high band gaps, giving rise to up-conversion, and the Ti-sapphire laser $E_{2}$ was scanned across the band gap of GaAs. The optical setup was arranged such that the beams were exactly colinear and excited the sample at the same position. The result of the experiment for sample 1 is given in Fig. 3; the dashed line shows the UPL intensity with only $E_{1}$ photons. It is seen that the UPL signal is not enhanced when $E_{2}$ is below the GaAs band gap. Instead, it increases when $E_{2}$ passes the GaAs band gap, ruling out the aforementioned interaction. The step enhancement in Fig. 3 is in perfect agreement with the generation mechanism by cold-Auger processes, the schematic of which is shown as an inset in Fig. 3.

In Fig. 4 the intensity of the UPL signal of sample 4 is given as a function of reciprocal temperature, along with that of the regular PL signal for which the excitation source was either an $\mathrm{Ar}^{+}$laser line at $514 \mathrm{~nm}$ or one at $488 \mathrm{~nm}$. The UPL signal decreases much less rapidly with temperature than the regular PL signal and it can still be observed at

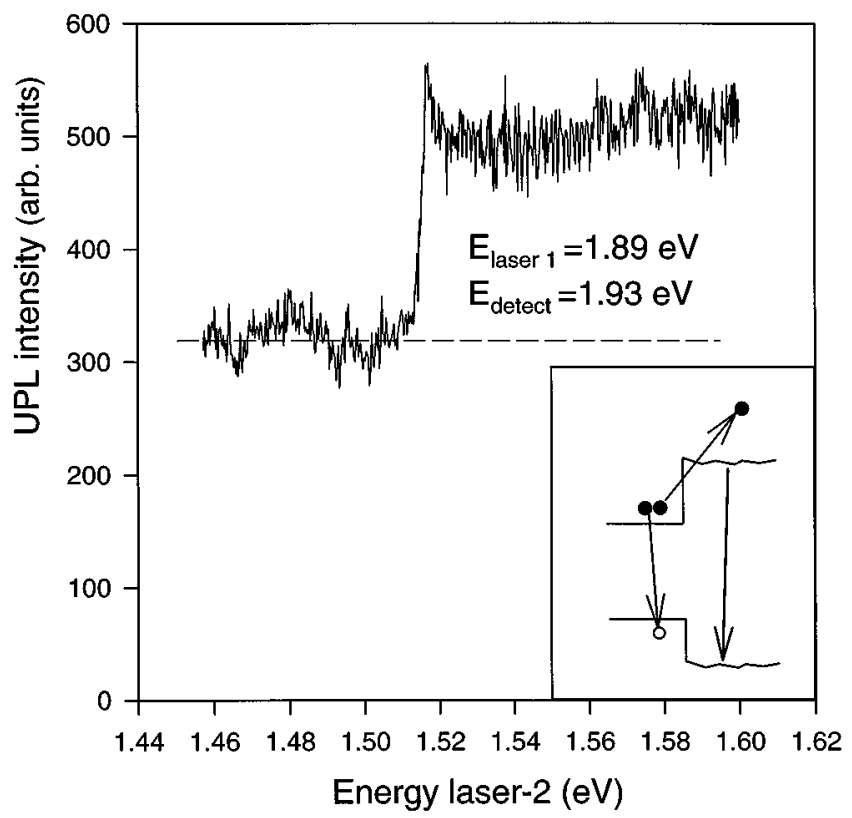

FIG. 3. Colinear double-beam experiment: the intensity of the UPL is monitored at $1.93 \mathrm{eV}$ with one laser exciting at $E_{1}=1.89$ $\mathrm{eV}$, and another laser $E_{2}$ being tuned between 1.46 and $1.60 \mathrm{eV}$. The inset shows a schematic of the cold (eeh) Auger process at the $\mathrm{GaAs} / \mathrm{Al}_{x} \mathrm{Ga}_{1-x} \mathrm{InP}_{2}$ interface [ehh processes occur in a similar way], carrier cooling and trapping, and PL from the $\mathrm{Al}_{x} \mathrm{Ga}_{1-x} \mathrm{InP}_{2}$.

$T=200 \mathrm{~K}$. The thermal behavior of the generation rate $G$ of Auger electrons (eeh process) is given by ${ }^{7}$

$$
G(\mathbf{k})=8 \pi^{2} \frac{E_{B}}{\hbar}\left[\frac{\hbar}{m_{e} E_{g}}\right]^{5} \frac{\hbar^{2} T}{m_{e}\left(\Delta E_{g}\right)^{2}} S n_{e}^{2} n_{h},
$$

where $E_{B}$ is the effective Rydberg, $E_{g}$ is the band gap of the lowest gap layer, $\Delta E_{g}$ is the band-gap difference (assuming symmetric offsets), $S$ is the surface area of the heterojunc-

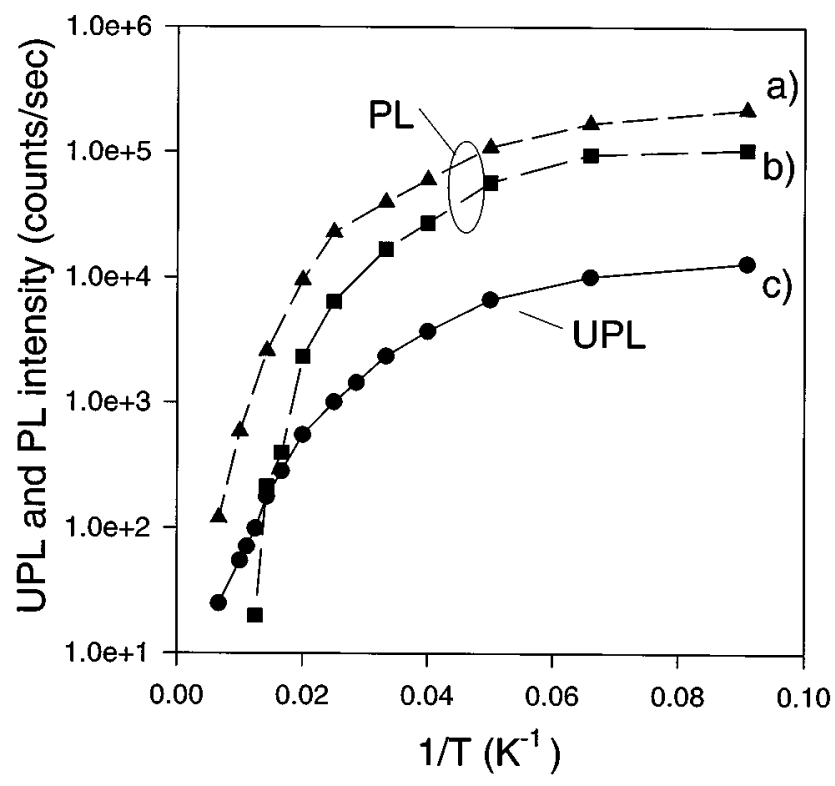

FIG. 4. Temperature dependence of the intensities of UPL and PL (for two different excitation wavelengths). 
tion, and $n_{e}$ and $n_{h}$ are the electron and hole densities, respectively. Predominantly as a result of thermal shrinkage of the band gap, this rate increases with increasing $T$, which may in part account for the temperature dependence of our data. However, in addition to the temperature dependence of the generation process, that of the PL emission process also should be taken into account. The latter will reflect thermal statistics of excitons or impurity states, and also diffusion of free carriers to the surface. That surface recombination plays a role can be observed by the differences in the curves obtained via 488 and $514 \mathrm{~nm}$ excitation. The 488 -nm laser light has a smaller penetration depth and free carriers will diffuse to the surface more easily; the corresponding curve in Fig. 4 indeed decreases slightly faster than that involving $514 \mathrm{~nm}$ excitation. For the UPL signal, diffusion to the surface can be neglected since these carriers are generated at the interface, a region which, for sample 4 with its $1.5-\mu \mathrm{m}$ $\mathrm{Al}_{x} \mathrm{Ga}_{1-x} \mathrm{InP}_{2}$ cap layer, is not affected by the surface depletion field. We emphasize that the behavior displayed in Fig. 4 does not need to be present in all junctions because, for instance, the presence of nonradiative traps at the interface with activation energies could lead to a faster decrease in the UPL intensity.

Efficient PL emission at energies up to $700 \mathrm{meV}$ above the laser excitation energy was observed at type-I

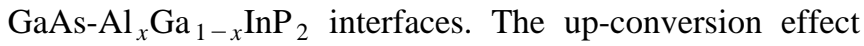
was shown to be induced by generation of electrons and holes in the low-gap semiconductor and attributed to a recently calculated cold interface-Auger process, which enables the up-conversion of both carrier types. The temperature dependence of the signal and the results from a colinear double-beam experiment showed agreement with this assignment. Furthermore, the presence of localized states in the barrier layer, induced by deviations from perfect random arrangements of the group-III atoms, was shown to be crucial in observing the up-conversion as a radiative transition.

E.D. Jones, A. Zunger, and Yong Zhang are gratefully acknowledged for stimulating discussions. This work was supported by U.S. Department of Energy Contract No. DEAC36-83CH10093, and by Stichting Technische Wetenschappen (STW) Contract No. NNS44.3510.
*Present address: Philips Semiconductors, Gerstweg 2, 6534 AE Nijmegen, The Netherlands.

${ }^{1}$ Light Scattering in Solids I; Introductory Concept, 2nd ed., edited by M. Cardona, Topics in Applied Physics, Vol. 8 (Springer, Berlin, 1983).

${ }^{2}$ E. Gornik, T. Y. Chang, T. J. Bridges, V. T. Nguyen, J. D. McGee, and W. Müller, Phys. Rev. Lett. 40, 1151 (1978).

${ }^{3}$ P. Vagos, P. Boucaud, F. H. Julien, J.-M. Lourtioz, and R. Planel, Phys. Rev. Lett. 70, 1018 (1993).

${ }^{4}$ E. J. Schubert and K. Ploog, J. Phys. C 18, 4549 (1985).

${ }^{5}$ M. Potemski, R. Stepniewski, J. C. Maan, G. Martinez, P. Wyder, and B. Etienne, Phys. Rev. Lett. 66, 2239 (1991).

${ }^{6}$ D. S. Kim, H. S. Ko, Y. M. Kim, S. J. Rhee, S. C. Hong, W. S. Kim, J. M. Chung, S. Huhr, and J. C. Woo, in Compound Semiconductors 1995, Insititute of Physics Conference Series 145 , edited by J. C. Woo and Y. S. Park (IOP, Bristol, 1996), p. 855.

${ }^{7}$ G. G. Zegrya and V. A. Kharchenko, Zh. Eksp. Teor. Fiz. 101, 327 (1992) [Sov. Phys. JETP 74, 173 (1992)].
${ }^{8}$ W. Seidel, A. Titkov, J. P. André, P. Voisin, and M. Voos, Phys. Rev. Lett. 73, 2356 (1994); A. Titkov, W. Seidel, J. P. André, P. Voisin, and M. Voos, Solid State Electron. 37, 1041 (1994).

${ }^{9}$ F. A. J. M. Driessen, Appl. Phys. Lett. 67, 2813 (1995).

${ }^{10}$ F. A. J. M. Driessen, H.M. Cheong, and A. Mascarenhas, in $O p$ toelectronic Materials-Ordering, Composition Modulation, and Self-Assembled Structures, MRS Symposia Proceedings No. 417, edited by E.D. Jones, A. Mascarenhas, and P. Petroff, (Materials Research Society, Pittsburgh, 1996), p. 85.

${ }^{11}$ P. Ernst, C. Geng, F. Scholz, H. Schweizer, Yong Zhang, and A. Mascarenhas, Appl. Phys. Lett. 67, 2347 (1995).

${ }^{12}$ K. A. Mäder and A. Zunger, Appl. Phys. Lett. 74, 2882 (1994).

${ }^{13}$ T. Kobayashi, K. Taira, F. Nakamura, and H. Kawai, J. Appl. Phys. 65, 4894 (1989); it should be noted that the reported conduction-band offsets for the $\mathrm{GaAs} / \mathrm{GaInP}_{2}$ junction in the literature scatter between 30 and $390 \mathrm{meV}$, whereas the valenceband offset scatters less and is approximately $250 \mathrm{meV}$. 